\title{
Abelian torsion groups with a pseudocompact group topology ${ }^{1}$
}

\author{
W.W. Comfort and Dieter Remus
}

(Communicated by Karl H. Hofmann)

\begin{abstract}
Two questions are posed: (a) Which Abelian torsion groups admit a PGT (pseudocompact group topology)? (b) If an Abelian torsion group $G$ admits a PGT, for which cardinal numbers $\alpha$ may such a topology $\mathscr{T}$ be chosen so that the weight of the space $\langle G, \mathscr{T}\rangle$ is equal to $\alpha$ ? The authors answer question (a) completely (Theorems 3.17 and 3.19). In Theorem 3.24 for $\alpha \geq \gamma \geq \omega$ they characterize those Abelian torsion groups of cardinality $\gamma$ which admit a PGT of weight $\alpha$. This furnishes partial answers to (b).
\end{abstract}

1991 Mathematics Subject Classification: 54H11, 20K45; 54A35, $22 \mathrm{~A} 05$.

\section{§ 0. Introduction}

A Tychonoff space $X$ is said to be pseudocompact if every continuous real-function on $X$ is bounded. As Hewitt [22] showed when he introduced the condition, it is equivalent to the condition that $X$ is $G_{\delta}$-dense in its Stone-Čech compactification $\beta X$ (in the sense that $X$ intersects every non-empty $G_{\delta^{-}}$-subset of $\beta X$ ).

Following Hewitt's work [22], independently and approximately simultaneously, Corson [12] and Glicksberg [20] and Kister [26] introduced pseudocompactness into the context of topological groups with the following result: If $\left\{X_{i}: i \in I\right\}$ is a set of compact (Tychonoff) spaces with product $K$, and if $p_{i} \in X_{i}$, then the $\Sigma$-product

$$
S=\left\{x \in K: \mid\left\{i \in I: x_{i} \neq p_{i} \mid \leq \omega\right\}\right.
$$

1 This work was completed in the summer of 1991 when the first-listed author enjoyed a "study-grant" from the Deutscher Akademischer Austauschdienst (="DAAD") of the Federal Republic of Germany and, subsequently, generous hospitality and support from the Technische Hochschule Darmstadt. He is pleased to thank these institutions, as well as the National University of Singapore for hospitality while the manuscript was prepared for publication (summer, 1992). 
is pseudocompact; furthermore, $K=\beta S$ in this case. (It is clear that $S$ is a topological group if each $X_{i}$ is a topological group and the point $p_{i}=e_{i}$ is selected.) Motivated in part by the observation that $S$ as above is $G_{\delta}$-dense in $K$, Comfort and Ross [11] initiated a formal study of pseudocompact groups (see 1.4 below for a statement of the principal results of [11]). Their results were subsequently strengthened and generalized by many workers, including M. Hušek, M. Tkachenko, J. Trigos-Arrieta, V. Uspenskií, and J. de Vries (see [3] for specific references) and more recently by Hernández and Sanchis [21]. The surveys [2] and [3] cite numerous additional investigations of pseudocompactness in the context of topological groups, many of them dealing with aspects of the (still unsolved) question whether every pseudocompact group of uncountable weight admits a proper dense pseudocompact subgroup.

While the topological theory of pseudocompact groups enjoys, then, a certain vitality, there exists at present no algebraic characterization of those groups which admit a pseudocompact group topology. (In contrast, the structure of those Abelian groups which admit a compact group topology has been understood for some decades; see Hewitt and Ross [23] (25.25) for a complete characterization.) In the present paper we solve this problem for Abelian torsion groups. Our characterization depends on the fact that every pseudocompact Abelian torsion group is of bounded order (Theorem 1.3), hence is algebraically the direct sum of finite cyclic groups (cf. [18] (17.2)). Theorem 3.19 allows us to reduce the problem to Abelian $p$-groups of bounded order, while 3.14 illustrates the difficulties encountered in the general case: let $\gamma$ be an inadmissible cardinal (cf. $\S 2$ ), fix a prime number $p$, and define $G_{0}=\bigoplus_{\gamma} \mathbb{Z}(p) \oplus \oplus_{2^{\gamma}} \mathbb{Z}\left(p^{2}\right)$, and $G_{1}=\bigoplus_{{ }^{\gamma}} \mathbb{Z}(p) \oplus \oplus_{\gamma} \mathbb{Z}\left(p^{2}\right)$; then $G_{0}$ admits a pseudocompact group topology, and $G_{1}$ does not. This example shows inter alia that the algebraic structure of the socle of a $p$-group $G$ does not determine whether $G$ admits a pseudocompact group topology.

Our characterization of those Abelian torsion groups which admit a pseudocompact group topology takes a particularly pleasing form (Theorem 3.20) in the axiom system $\mathrm{ZFC}+(\mathrm{M})$. (Here axiom (M), stated in 2.4 below, is a consequence of the singular cardinals hypothesis. Whether (M) is a theorem of ZFC is unknown; see 2.4 for a discussion of this point.)

We characterize in Theorem 3.24 all infinite Abelian torsion groups admitting a pseudocompact group topology of weight $\alpha$ with $|G| \leq \alpha$, and en route to our principal results we show Theorem 3.5: for infinite cardinals $\gamma$ and $\alpha$ and for finite Abelian groups $F$ with $|F|>1$, if some group of cardinality $\gamma$ admits a pseudocompact group topology of weight $\alpha$, then $\bigoplus_{\gamma} F$ does so; and in this case, the choice $\alpha=\log \gamma$ is possible.

This paper is a sequel to our earlier work [7]. Some results similar to ours have been achieved independently and approximately simultaneously, and announced without proof, by Dikranjan and Shakhmatov [14]. Our own announcements appear in [5], [6], and [3] (\$3.10B).

0.1. Acknowledgement. We thank Professor K.H. Hofmann for extensive helpful comments. 


\section{§1. Preliminaries}

We consider only completely regular, Hausdorff spaces. The cardinality of a set $X$ is denoted $|X|$. We denote the identity element of the groups we consider (whether or not assumed Abelian) by the symbol 0 .

The weight and density character of a space $X$ are written $w(X)$ and $d(X)$, respectively.

The following three statements will be used below.

1.1. Theorem. Let $G$ be a dense subgroup of a topological group $K$.

(a) If $\mathscr{B}$ is a local base for $K$ at 0 , then $\{x B: x \in G, B \in \mathscr{B}\}$ is a base for $K$.

(b) $w(G)=w(K)$.

Proof. (a) is easily proved, as is (b) in case $|G|<\omega$; so we take $|G| \geq \omega$. The "local weight function" $\chi$ satisfies $\chi(0, G)=\chi(0, K)$, so (a) gives

$$
w(K) \leq \chi(K) \cdot d(K) \leq \chi(G) \cdot d(G) \leq w(G) \cdot w(G)=w(G),
$$

as required.

1.2. Theorem. (a) The continuous image of a pseudocompact space is pseudocompact.

(b) Let $\langle G, \mathscr{T}\rangle$ be an infinite pseudocompact Abelian group. Then there is a pseudocompact group topology $\mathscr{U}$ on $G$ such that $\mathscr{U} \subseteq \mathscr{T}$ and $w(G, \mathscr{U}) \leq|G|$.

Proof. (a) is obvious, as is (b) in case $w(G, \mathscr{T}) \leq|G|$. We assume then that $w(G, \mathscr{T})>|G|$. According to $1.1(\mathrm{~b})$ and $[23](24.15,22.17)$, the Pontrjagin dual $\hat{K}$ of the compact completion $K$ of $(G, \mathscr{T})$ satisfies (1) $|\hat{K}|=w K=w(G, \mathscr{T})$ and (2) $\hat{K}$ separates points of $K$. Clearly there is a subgroup $H$ of $\hat{K}$ such that $|H| \leq|G|$ and $\{h \mid G: h \in H\}$ separates points of $G$. The (Hausdorff) topology $\mathscr{U}$ on $G$ induced by $\{h \mid G: h \in H\}$ satisfies $\mathscr{U} \subseteq \mathscr{T}$ and $w(G, \mathscr{U}) \leq|H| \leq|G|$, and $\mathscr{U}$ is pseudocompact by part (a).

The proof of 1.2(b) is a special case of a construction given in [10]. It follows from [10], [23] (24.15) and 1.1 that $w(G, \mathscr{U})=|H|$.

For compact Abelian groups, 1.3 is a consequence of the Baire category theorem noted in [23] (25.9); the generalization to the pseudocompact case, given in [9] (7.4), is routine.

1.3. Theorem ([9]). Every pseudocompact Abelian torsion group is of bounded order.

A topological group $G=\langle G, \mathscr{T}\rangle$ is said to be totally bounded (by some authors: pre-compact) if for every non-empty $U \in \mathscr{T}$ there is a finite $F \subseteq G$ such that $G=F U$. 
It is a theorem of Weil [31] that there is a compact group $\bar{G}$, unique in an obvious sense, in which $G$ is dense; we call $\bar{G}$ the Weil completion of $G$.

\subsection{Theorem ([11]). (a) Every pseudocompact group is totally bounded;}

(b) a totally bounded group $G$ is pseudocompact if and only if $G$ is $G_{\delta^{-}}$-dense in its Weil completion; and

(c) if $\left\{G_{i}: i \in I\right\}$ is a set of pseudocompact groups, then $\prod_{i \in I} G_{i}$ is pseudocompact.

The expression $G \approx H$ indicates that $G$ and $H$ are isomorphic groups; the ambient topological structures (if any) are ignored.

The symbols $\alpha, \beta, \gamma$, and $\kappa$ denote cardinal numbers, and $\omega$ is the least infinite cardinal. The symbol $\xi$ denotes an ordinal number.

For $\alpha \geq \omega$ we write $\log \alpha=\min \left\{\beta: 2^{\beta} \geq \alpha\right\}$. Clearly $\alpha=\log \alpha$ if and only if every $\beta<\alpha$ satisfies $2^{\beta}<\alpha$; such a cardinal $\alpha$ is called a strong limit cardinal.

The symbols $\mathbb{N}$ and $\mathbb{P}$ denote respectively the set of positive integers and the set of prime numbers; and for $n \in \mathbb{N}$ we write $\mathbb{Z}(n)$ for the cyclic group of order $n$.

\section{§ 2. The "Function" $m$ and Hypothesis (M)}

We begin this section with a definition from [8].

2.1. Definition. For each compact group $K$, define

$$
m(K)=\min \{|G|: G \text { is a dense, pseudocompact subgroup of } K\} .
$$

It is known [8] that $m(K)$ depends only on $w(K)$ - that is, if $K$ and $K^{\prime}$ are compact groups with $w(K)=w\left(K^{\prime}\right)$ then $m(K)=m\left(K^{\prime}\right)$. Hence for $\alpha \geq \omega$ one may define $m(\alpha)$ to be $m(K)$ for some (equivalently: every) compact group $K$ such that $w(K)=\alpha$. According to the criterion cited in 1.4(b), we have $m(\alpha)=d(P(K))$ for each such $K$. (Here for a space $X$ the symbol $P(X)$ denotes the set $X$ with the topology generated by the $G_{\delta}$-subsets of the space $X$. Standard information about spaces of the form $P(X)$ is available, for instance, in $[4](\S 2)$. Thus in the interest of specificity one might as well define $m(\alpha)$ by the rule

$$
m(\alpha)=d\left(P\left(\{0,1\}^{\alpha}\right)\right) .
$$

With $m(\alpha)$ so defined, the following statement is transparent.

2.2. Theorem ([7]). Let $\omega \leq \alpha \leq \beta$. Then $m(\alpha) \leq m(\beta)$.

The following statement summarizes most of the (other) information known about the cardinal numbers $m(\alpha)$.

2.3. Theorem ([1], [8]). Let $\alpha \geq \omega$. Then
(a) $m(\alpha) \geq \mathbf{c}$;
(b) $\operatorname{cf}(m(\alpha))>\omega$;
(c) $\log \alpha \leq m(\alpha) \leq(\log \alpha)^{\omega}$. 


\subsection{Discussion. In [7], the statement}

$$
\text { " } m(\alpha)=(\log \alpha)^{\omega} \quad \text { for all } \alpha \geq \omega ",
$$

suggested by $2.3(\mathrm{c})$, is denoted $(\mathrm{M})$. Whether $(\mathrm{M})$ is a theorem of $\mathrm{ZFC}$ is a question raised in [1] and in [8]. It is known [1], [8] that the singular cardinals hypothesis (here abbreviated $\mathrm{SCH}$ ) implies (M). Since $\mathrm{SCH}$ is equivalent to the condition that $\kappa^{\omega}=\kappa$ for all $\kappa \geq \mathbf{c}$ with $\mathrm{cf}(\kappa)>\omega$ (cf. Jech [25] (§ 8)), while (according to 2.3) (M) is equivalent to the condition that $\kappa^{\omega}=\kappa$ for cardinals of the form $\kappa=m(\alpha)$, it is natural to inquire whether $(\mathrm{M}) \Rightarrow \mathrm{SCH}$. Assuming the consistency relative to $\mathrm{ZFC}$ of suitable large cardinal axioms, this implication cannot be proved, since Masaveu [30] has shown that (M) holds in certain of the models of Magidor [28], [29] in which SCH fails. (Devlin and Jensen [13] have shown that some large cardinal assumption is necessary for the construction of a model where $\mathrm{SCH}$ fails.) Masaveu's calculations keep alive the possibility that the answer to the following question, reiterated here in the interest of completeness, is "Yes." We believe however that (M) fails in certain models of ZFC.

\subsection{Question [1], [8]. Is (M) a theorem of ZFC?}

As in [7], we denote by $\mathscr{G}(\alpha)$ the class of groups which admit a pseudocompact group topology of weight $\alpha$; appropriating a term from [14] we say that a cardinal $\gamma$ is admissible if some group of cardinality $\gamma$ admits a pseudocompact group topology. (According to this use of the term, the positive finite cardinals are admissible. Though we have little use for the finite cardinals in this context, this convention simplifies the statement of some of our theorems and is otherwise harmless.) Using different notation and terminology, the infinite admissible cardinals were characterized in the axiom system $\mathrm{ZFC}+\mathrm{SCH}$ (in terms of their arithmetic properties) by van Douwen [17]; see [8] for a more full account, and see [7] for proofs in succinct language of several of the results of [17]. In any event it is clear that for infinite cardinals $\alpha$ and $\gamma$ the following two conditions are equivalent: $m(\alpha) \leq \gamma \leq 2^{\alpha}$; some group $G$ with $|G|=\gamma$ satisfies $G \in \mathscr{G}(\alpha)$. It should be noted explicitly however that not every group $G$ such that $|G|$ is admissible admits a pseudocompact group topology. Isolated examples are given in [7], and the existence of large families of such groups $G$ will become apparent in Theorems 3.17 and 3.19 below.

When a shift of emphasis is appropriate, we write $\alpha \in \mathscr{A}(\gamma)$ in place of $m(\alpha) \leq \gamma \leq 2^{\alpha}$.

\section{§ 3. Finding some pseudocompactifiable groups}

3.1. Lemma. Let $\alpha \geq \omega$ and let $F$ be a finite Abelian group with $|F|>1$. Then $F^{\alpha} \approx \bigoplus_{2^{\alpha}} F$. 
Proof. It is enough to treat the case $F=\mathbb{Z}\left(p^{r}\right)$ with $p \in \mathbb{P}, 0<r<\omega$; for if the result is known for such groups then from the isomorphism $F \approx \bigoplus_{i=1}^{n} \mathbb{Z}\left(p_{i}^{r_{i}}\right)=\prod_{i=1}^{n} \mathbb{Z}\left(p_{i}^{r_{i}}\right)$ will follow

$$
\begin{aligned}
F^{\alpha} & \approx\left(\prod_{i=1}^{n} \mathbb{Z}\left(p_{i}^{r_{i}}\right)\right)^{\alpha}=\prod_{i=1}^{n}\left(\mathbb{Z}\left(p_{i}^{r_{i}}\right)\right)^{\alpha} \\
& \approx \prod_{i=1}^{n} \bigoplus_{2^{\alpha}} \mathbb{Z}\left(p_{i}^{r_{i}}\right)=\bigoplus_{2^{\alpha}}\left[\prod_{i=1}^{n} \mathbb{Z}\left(p_{i}^{r_{i}}\right)\right]=\bigoplus_{2^{\alpha}} F .
\end{aligned}
$$

The case $F=\mathbb{Z}\left(p^{r}\right)$ is not difficult to prove; see Hewitt and Ross [24] (Lemma 4.5).

3.2. Theorem. Let $\alpha$ and $\gamma$ be infinite cardinals and let $F$ be a finite Abelian group with $|F|>1$. If $F^{\alpha}$ contains $a G_{\delta}$-dense subset of cardinality $\gamma$ then $F^{\alpha}$ contains $a G_{\delta}$-dense copy of $\oplus_{\gamma} F$.

Proof. Let $\psi: F^{\alpha} \rightarrow \bigoplus_{2^{\alpha}} F$ be an isomorphism guaranteed by 3.1, let $D$ be a $G_{\delta}$-dense subset of $F^{\alpha}$ with $|D|=\gamma$, and set

$$
A=\left\{\xi<2^{\alpha}: \pi_{\xi}[\psi[D]] \neq\{0\}\right\}
$$

with $\pi_{\xi}: \bigoplus_{2^{\alpha}} F \rightarrow F_{\xi}$ the canonical projection. From $\gamma \geq \omega$ and $|F|<\omega$ follows $|A|=\gamma$, so $\bigoplus_{\gamma} F \approx \psi^{-1}\left(\bigoplus_{\xi \in A} F_{\xi}\right) \subseteq F^{\alpha}$. From $D \subseteq \psi^{-1}\left(\bigoplus_{\xi \in A} F_{\xi}\right)$ it follows that $\psi^{-1}\left(\bigoplus_{\xi \in A} F_{\xi}\right)$ is $G_{\delta}$-dense in $F^{\alpha}$, as required.

It is clear for $F$ and $\alpha$ as above that $w\left(F^{\alpha}\right)=\alpha$. It then follows from 1.1 and 1.4(b) that 3.2 may be recast as follows.

3.3. Theorem. Let $\alpha$ and $\gamma$ be infinite cardinals and let $F$ be a finite Abelian group with $|F|>1$. Then the following statements are equivalent.

(a) $m(\alpha) \leq \gamma \leq 2^{\alpha}$;

(b) $\bigoplus_{\gamma} F \in \mathscr{G}(\alpha)$.

If a group happens to admit a pseudocompact group topology, there may be various such topologies of different weights. The following result is suggestive.

3.4. Lemma. Every infinite admissible cardinal $\gamma$ satisfies $\log \gamma \in \mathscr{A}(\gamma)$.

Proof. There is $\alpha \geq \omega$ such that $m(\alpha) \leq \gamma \leq 2^{\alpha}$, so $m(\log \gamma) \leq m(\alpha) \leq \gamma$ follows from $\log \gamma \leq \alpha$ and 2.2. That $\gamma \leq 2^{\log \gamma}$ is evident.

With the help of 3.4, Theorem 3.3 takes the following form. 
3.5. Theorem. Let $\gamma$ be an infinite cardinal and let $F$ be a finite Abelian group with $|F|>1$. Then the following statements are equivalent.

(a) $\gamma$ is admissible;

(b) there is $\alpha$ such that $\bigoplus_{\gamma} F \in \mathscr{G}(\alpha)$; and

(c) $\bigoplus_{\gamma} F \in \mathscr{G}(\log \gamma)$.

3.6. Remark. Taken together, Theorems 3.3 and 3.5 show that the groups $\bigoplus_{\gamma} F$ serve as "test spaces" for the admissibility of the cardinal $\gamma$ : If any group of cardinality $\gamma$ admits a pseudocompact group topology of weight $\alpha$, then $\bigoplus_{\gamma} F$ does so; and in this case $\bigoplus_{\gamma} F$ admits a pseudocompact group topology of weight $\log \gamma$. In our work [7], by different methods we have achieved identical results for the groups $\oplus_{\gamma} \mathbb{Q}$ and $\oplus_{\gamma} \mathbb{Z}$ - but in the latter case only in the axiom system ZFC $+(\mathrm{M})$ for $\alpha>\omega$. The fact that $\oplus_{\gamma} \mathbb{Z}$ serves (in this sense) as a test space for the admissibility of the cardinal $\gamma$ has been announced in ZFC alone, with no additional axioms, by Dikranjan and Shakhmatov (see [3] (3.10 B.2)).

3.7. Question. Let $G$ be a group which admits a pseudocompact group topology - that is, $G \in \mathscr{G}(\kappa)$ for some cardinal $\kappa$. Suppose further that $\alpha \in \mathscr{A}(|G|)$ with $\alpha>\omega$. Must $G \in \mathscr{G}(\alpha)$ ? What about the special case $\alpha=\log |G|$ ?

We ask a sharpened version of 3.7 in 3.22 below. Although we have been unable to settle Question 3.7 even when $G$ is an Abelian torsion group, we have successfully determined which Abelian torsion groups do admit a pseudocompact group topology. We turn now to that subject, beginning with a useful characterization of the admissible cardinals.

\subsection{Theorem. Let $\gamma$ be an infinite cardinal.}

(a) $\gamma$ is admissible if and only if the compact group $\{0,1\}^{\log \gamma}$ admits a dense pseudocompact subgroup of cardinality $\gamma$.

(b) Assume (M). Then $\gamma$ is admissible if and only if $\gamma \geq(\log \log \gamma)^{\omega}$.

(c) Assume SCH. Then $\gamma$ is admissible if and only if $\gamma \geq(\log \gamma)^{\omega}$.

Proof. From 3.4 the cardinal $\gamma$ is admissible if and only if $\gamma \geq m(\log \gamma)$, so (a) and (b) are clear.

(c) From SCH and $\gamma \geq(\log \gamma)^{\omega}$ follow (M) and $\gamma \geq(\log \log \gamma)^{\omega}$, so $\gamma$ is admissible by (b). For the converse, assume that $\gamma$ is admissible. If $\gamma=\log \gamma$ then $\gamma \geq(\log \gamma)^{\omega}$ by (b), and if $\gamma>\log \gamma$ then from SCH and $\operatorname{cf}\left((\log \gamma)^{+}\right)>\omega$ follows

$$
\gamma \geq(\log \gamma)^{+}=\left((\log \gamma)^{+}\right)^{\omega} \geq(\log \gamma)^{\omega} .
$$


It occurs for many cardinals $\gamma$ that $\gamma=2^{\log \gamma}$. (Every cardinal $\gamma$ of the form $\gamma=2^{\alpha}$ satisfies that relation.) Clearly, such $\gamma$ satisfy the condition of Theorem 3.5. Although strong limit cardinals $\gamma$ never satisfy $\gamma=2^{\log \gamma}$, it is nevertheless easy to determine which limit cardinals are, and which are not, admissible.

3.9. Theorem (cf. also [17]). Let $\gamma$ be a strong limit cardinal. Then $\gamma$ is admissible if and only if $\operatorname{cf}(\gamma)>\omega$.

Proof. If $\gamma$ is admissible then from 3.4 and 2.3(c) follows

$$
\gamma=\log \log \gamma \leq m(\log \gamma) \leq \gamma ;
$$

thus $\gamma=m(\log \gamma)$, and $\operatorname{cf}(\gamma)>\omega$ follows from 2.3(b).

Conversely if $\operatorname{cf}(\gamma)>\omega$ then again from 2.3 follows

$$
m(\log \gamma) \leq(\log \log \gamma)^{\omega}=\gamma^{\omega}=\sum_{\delta<\gamma} \delta^{\omega} \leq \sum_{\delta<\gamma} 2^{\delta}=\gamma \leq 2^{\log \gamma},
$$

so $\log \gamma \in \mathscr{A}(\gamma)$.

The following lemma, which shows that for every infinite admissible cardinal $\gamma$ there exists $\alpha$ such that $m(\alpha) \leq \gamma<2^{\alpha}$, will allow us to extend (in part) the results of 3.3 to groups of the form $\oplus_{\gamma} F \oplus \oplus_{i<\gamma} G_{i}$ with each $G_{i} \subseteq F$. Our notation is intended to admit the possibility that $G_{i}=\{0\}$ for some or all of the groups $G_{i}$, so 3.11 and 3.12 apply in particular to the groups $\oplus_{\gamma} F$.

3.10. Lemma. Let $\alpha$ and $\gamma$ be infinite cardinals such that $\alpha \in \mathscr{A}(\gamma)$. Then either

(i) $m(\alpha)<2^{\alpha}$, or

(ii) $\left(m(\alpha)=\gamma=2^{\alpha}\right.$ and) every cardinal $\kappa$ such that $\gamma \leq \kappa \leq 2^{\gamma}$ satisfies $\kappa \in \mathscr{A}(\gamma)$ and $m(\kappa)<2^{\kappa}$.

Proof. Suppose that (i) fails, and let $\gamma \leq \kappa \leq 2^{\gamma}$. Then from 2.2 follows

$$
m(\kappa) \leq m\left(2^{\gamma}\right) \leq\left(\log \left(2^{\gamma}\right)\right)^{\omega} \leq \gamma^{\omega}=\gamma \leq \kappa<2^{\kappa},
$$

so (ii) holds.

3.11. Theorem. Let $\alpha \in \mathscr{A}(\gamma)$ with $\gamma \geq \omega$, let $F$ be a finite Abelian group with $|F|>1$, and let $G=\bigoplus_{\gamma} F \oplus \bigoplus_{i<\gamma} G_{i}$ with each $G_{i}$ a subgroup of $F$. Then either

(i) $G \in \mathscr{G}(\alpha)$, or

(ii) $\gamma=2^{\alpha}$ and $G \in \mathscr{G}(\kappa)$ for every $\kappa$ such that $\gamma \leq \kappa \leq 2^{\gamma}$.

Proof. If $m(\alpha)<2^{\alpha}$ let $\kappa=\alpha$ in what follows, and otherwise let $\kappa$ be as in (ii). Then from 3.10 follows $\kappa \in \mathscr{A}(\gamma)$ and $m(\kappa)<2^{\kappa}$. Now from Definition 2.1 and Theorem 1.4(b), there is a $G_{\delta}$-dense subgroup $N$ of $F^{\kappa}$ such that $|N|=m(\kappa)$. Let 
$\psi: F^{\kappa} \rightarrow \bigoplus_{2^{\kappa}} F$ be an isomorphism (as given by 3.1), let $H=\psi[N]$, and as in the proof of Theorem 3.2 set $A=\left\{\xi<2^{\kappa}: \pi_{\xi}[H] \neq\{0\}\right\}$ with $\pi_{\xi}: \bigoplus_{2^{\kappa}} F \rightarrow F_{\xi}$ the projection mapping. Then $|A|=m(\kappa)$ and $H \subseteq \bigoplus_{\xi \in A} F_{\xi} \subseteq \bigoplus_{2^{\kappa}} F_{\xi}$ (with each $F_{\xi}=F$ ). Since $|A|<2^{\kappa}$ and $|A| \leq \gamma \leq 2^{\kappa}$, there are disjoint subsets $B$ and $C$ of $2^{\kappa}$ such that $A \subseteq B$ and $|B|=|C|=\gamma$. The group $\bigoplus_{\xi \in C} F_{\xi}$ contains an isomorph $S$ of $\bigoplus_{i<\gamma} G_{i}$, and then the group $G^{\prime}:=\bigoplus_{\xi \in B} F_{\xi} \oplus S$ satisfies $G^{\prime} \approx G$ and $H \subseteq \bigoplus_{\xi \in A} F_{\xi} \subseteq G^{\prime} \subseteq \bigoplus_{\xi<2^{\kappa}} F_{\xi}$. Since $N=\psi^{-1}(H)$ is $G_{\delta}$-dense in $F^{\kappa}$, the same is true of $\psi^{-1}\left(G^{\prime}\right)$ (an isomorph of $G$ ). Now 1.1 and 1.4(b) complete the proof.

3.12. Corollary. Let $\gamma$ be an infinite admissible cardinal, let $F$ be a finite Abelian group with $|F|>1$, and let $G=\bigoplus_{\gamma} F \oplus \bigoplus_{i<\gamma} G_{i}$ with each $G_{i}$ a subgroup of $F$. Then either

(i) $G \in \mathscr{G}(\log \gamma)$, or

(ii) $\gamma=2^{\log \gamma}$ and $G \in \mathscr{G}(\kappa)$ for every $\kappa$ such that $\gamma \leq \kappa \leq 2^{\gamma}$.

Proof. Lemma 3.4 gives $\log \gamma \in \mathscr{A}(\gamma)$, so this statement is the special case $\alpha=\log \gamma$ of 3.11 .

3.13. Remark. With $\alpha=\log \gamma$ the condition $\gamma<2^{\alpha}$ is sufficient, but not necessary, to ensure that a group $G$ of the form considered in 3.11 satisfies $G \in \mathscr{G}(\alpha)$. For an example to this effect let $\gamma=2^{\alpha}$ with $\alpha=\log \gamma$ and take $G=\bigoplus_{\gamma} F$ with $F$ a finite Abelian group with $|F|>1$. That $G \in \mathscr{G}(\alpha)$ is clear from the isomorphism $G=\bigoplus_{2^{\kappa}} F \approx F^{\alpha}$ of 3.1.

En route to the classification of those Abelian torsion groups which admit a pseudocompact group topology, we first consider $p$-groups. We treat the general case fully in 3.19; the following example, included as an aid to the reader, is intended to illustrate the principles and the difficulties encountered in the general case.

3.14. Theorem. Let $\gamma$ be an inadmissible cardinal, let $p \in \mathbb{P}$, and define

$$
G_{0}=\bigoplus_{\gamma} \mathbb{Z}(p) \oplus \bigoplus_{2^{\gamma}} \mathbb{Z}\left(p^{2}\right) \text {, and } G_{1}=\bigoplus_{2^{\gamma}} \mathbb{Z}(p) \oplus \bigoplus_{\gamma} \mathbb{Z}\left(p^{2}\right) \text {. }
$$

Then

(a) $G_{0}$ admits a pseudocompact group topology, and

(b) $G_{1}$ does not admit a pseudocompact group topology.

Proof. (a) is clear from what has preceded. (For example: $2^{\gamma}=2^{\log \left(2^{\gamma}\right)}$, so 3.11 (ii) ensures $G_{0} \in \mathscr{G}(\alpha)$ for every $\alpha$ such that $2^{\gamma} \leq \alpha \leq 2^{2^{\gamma}}$.)

(b) The image of $G_{1}$ under the map $x \rightarrow p x$ is the group $\bigoplus_{\gamma} \mathbb{Z}(p)$, whose cardinality is inadmissible. Since the continuous image of a pseudocompact space is pseudocompact, evidently the group $G_{1}$ has no pseudocompact group topology. 
3.15. Definition. A (possibly finite) sequence $\gamma_{1}, \gamma_{2}, \ldots, \gamma_{k} \ldots$ of cardinal numbers satisfies the cofinal admissibility condition if for every $k$ with $\gamma_{k}$ inadmissible there is $i>k$ such that $\gamma_{i}>\gamma_{k}$ and $\gamma_{i}$ is admissible.

The following algebraic result summarizes what we need to know of the structure of Abelian $p$-groups of bounded order. (As usual, the socle of a $p$-group is the kernel of the homomorphism $x \rightarrow p x$.) The first part of 3.16 is standard (cf. [18] (17.2); hints to a proof of the second part are given in [18] (17.4) and [27] (§ 24).

3.16. Theorem. For every Abelian p-group $G$ of bounded order, there exist an integer $n>0$, distinct positive integers $r_{i}(1 \leq i \leq n)$, and cardinal numbers $\gamma_{i}(1 \leq i \leq n)$, such that

$$
G=\bigoplus_{i=1}^{n} \bigoplus_{\gamma_{i}} \mathbb{Z}\left(p^{r_{i}}\right)
$$

The presentation (*) is unique up to isomorphism if the condition $r_{1}<r_{2}<\cdots<r_{n}$ is imposed. In this case the cardinal numbers $\gamma_{i}$ are determined by this rule: $\gamma_{i}$ is the rank of the quotient group $S \cap p^{r_{i}-1} G / S \cap p^{r_{i}} G$, with $S$ the socle of $G$.

In what follows we refer to (*) (with $r_{1}<r_{2}<\cdots<r_{n}$ ) as the canonical presentation of $G$. We say that $G$ satisfies the cofinal admissibility condition if the cardinal numbers $\gamma_{i}(1 \leq i \leq n)$ in the canonical presentation of $G$ satisfy the cofinal admissibility condition of Definition 3.15.

It is clear that if $G$ has canonical presentation $G=\bigoplus_{i=1}^{n} \bigoplus_{\gamma_{i}} \mathbb{Z}\left(p^{r_{i}}\right)$ and $G$ satisfies the cofinal admissibility condition, then $\gamma_{n}$ is admissible and $\max \left\{\gamma_{i}: 1 \leq i \leq n\right\}$ is admissible.

3.17. Theorem. Let $p \in \mathbb{P}$ and let $G$ be an Abelian p-group. Then the following conditions are equivalent.

(a) G admits a pseudocompact group topology.

(b) $G$ is of bounded order and $G$ satisfies the cofinal admissibility condition.

Proof. (a) $\Rightarrow$ (b). That $G$ is of bounded order is given by 1.3. Suppose now that $G=\bigoplus_{i=1}^{n} \bigoplus_{\gamma_{i}} \mathbb{Z}\left(p^{r_{i}}\right)$ with $r_{1}<\ldots<r_{n}$ but there is $k \leq n$ such that every $i$ with $k \leq i \leq n$ has either $\gamma_{i}<\gamma_{k}$ or $\gamma_{i}$ inadmissible. If $k=1$ then $|G|$ is the inadmissible cardinal $\gamma_{1}$, a contradiction, so we assume $k>1$. The continuous homomorphism $x \rightarrow p^{r_{k-1}} x$ from $G$ into $G$ has kernel $K$ such that $G / K=\bigoplus_{i=k}^{n} \bigoplus_{\gamma_{i}} \mathbb{Z}\left(p^{r_{i}-r_{k-1}}\right)$. Evidently $G / K$ admits a pseudocompact group topology (the quotient topology), yet $|G / K|=\sum_{i=k}^{n} \gamma_{i}$ is inadmissible.

(b) $\Rightarrow$ (a). We write $G=\bigoplus_{i=1}^{n} \bigoplus_{\gamma_{i}} \mathbb{Z}\left(p^{r_{i}}\right)$ with $r_{1}<\cdots<r_{n}$ and we argue by induction on $n$.

When $n=1$ we have $G=\bigoplus_{\gamma_{i}} \mathbb{Z}\left(p^{r_{1}}\right)$ with $\gamma_{1}$ admissible, and the required conclusion follows from 3.3. 
Suppose the statement true for $n \leq m$, and let $G=\bigoplus_{i=1}^{m+1} \bigoplus_{\gamma_{i}} \mathbb{Z}\left(p^{r_{i}}\right)$ with $\left\{\gamma_{i}: 1 \leq i \leq m+1\right\}$ as hypothesized. Let $i_{0}$ be the largest integer such that $1 \leq i_{0} \leq m+1$ and $\gamma_{i_{0}}=\max \left\{\gamma_{i}: 1 \leq i \leq m+1\right\}$, and note that $\gamma_{i_{0}}$ is admissible. If $i_{0}=m+1$ then $G$ has the form $G=\bigoplus_{\gamma} F \oplus \bigoplus_{i<\gamma} G_{i}$ with $\gamma=\gamma_{m+1}, F=\mathbb{Z}\left(p^{r_{m+1}}\right)$, and each $G_{i} \subseteq F$, so 3.11 appplies. If $i_{0}<m+1$ we write $G^{\prime}=\bigoplus_{i=1}^{i_{0}} \bigoplus_{\gamma_{i}} \mathbb{Z}\left(p^{r_{i}}\right)$ and $G^{\prime \prime}=\bigoplus_{i=i_{0}+1}^{m+1} \bigoplus_{\gamma_{i}} \mathbb{Z}\left(p^{r_{i}}\right)$ and then, noticing that $G^{\prime}$ and $G^{\prime \prime}$ satisfy the cofinal admissibility condition, we use the inductive hypothesis to find pseudocompact group topologies on $G^{\prime}$ and $G^{\prime \prime}$. The product topology on $G=G^{\prime} \times G^{\prime \prime}$ is then pseudocompact, as required.

Now using (M) we can rewrite 3.17, casting condition (b) only in the language of algebra and cardinal numbers.

3.18. Theorem. Assume (M). Let $p \in \mathbb{P}$ and let $G$ be an Abelian p-group. Then the following conditions are equivalent.

(a) G admits a pseudocompact group topology.

(b) $G$ has the form $G=\bigoplus_{i=1}^{n} \bigoplus_{\gamma_{i}} \mathbb{Z}\left(p^{r_{i}}\right)$ with $r_{1}<\cdots<r_{n}$ and with the family $\left\{\gamma_{i}: 1 \leq i \leq n\right\}$ satisfying these conditions: (1) either $\gamma_{n}$ is finite or $\left(\log \log \gamma_{n}\right)^{\omega} \leq \gamma_{n}$, and (2) for every $k$ such that $1 \leq k<n$ and $\omega \leq \gamma_{k}<\left(\log \log \gamma_{k}\right)^{\omega}$, there is $i$ such that $k<i \leq n$ and $\gamma_{i}>\gamma_{k}$ and $\left(\log \log \gamma_{i}\right)^{\omega} \leq \gamma_{i}$.

Proof. This is simply 3.17, incorporating 3.8(b).

3.19. Theorem. Let $G$ be an Abelian torsion group, and let $G=\bigoplus_{p \in \mathbb{P}} G(p)$ be the decomposition of $G$ into its p-components. Then the following conditions are equivalent.

(a) G admits a pseudocompact group topology;

(b) $G$ is of bounded order, and each of the groups $G(p)$ admits a pseudocompact group topology;

(c) $G$ is of bounded order, and each of the groups $G(p)$ satisfies the cofinal admissibility condition.

Proof. Since (b) $\Leftrightarrow$ (c) is given by 3.17 , and (b) $\Rightarrow$ (a) since the product of pseudocompact groups is pseudocompact, it is enough to prove (a) $\Rightarrow(b)$. Since $G$ is of bounded order by 1.3 , we have $G=\bigoplus_{j=1}^{m} G\left(p_{j}\right)$. For $1 \leq j \leq m$ let $n_{j}$ be the least integer such that $n_{j} x=0$ for each $x \in G\left(p_{j}\right)$, and set $n=\prod_{j=1}^{m} n_{j}$. The continuous homomorphism $x \rightarrow \frac{n}{n_{j}} x$ takes $G$ onto $G\left(p_{j}\right)$, so $G\left(p_{j}\right)$ is pseudocompact in the group topology inherited from $G$.

The combination of 3.18 and 3.19 yields this definitive formulation. 
3.20. Theorem. Assume (M). For an Abelian torsion group $G$, the following conditions are equivalent.

(a) G admits a pseudocompact group topology.

(b) $G$ has the form $G=\bigoplus_{j=1}^{m} \bigoplus_{i=1}^{n_{j}} \bigoplus_{\gamma_{j, i}} \mathbb{Z}\left(p_{j}^{r_{j, i}}\right)$ with $r_{j, 1}<\cdots<r_{j, n_{j}}$ for $1 \leq j \leq m$ and with each family $\left\{\gamma_{j, i}: 1 \leq i \leq n_{j}\right\}(1 \leq j \leq m)$ satisfying these conditions: (1) either $\gamma_{j, n_{j}}$ is finite or $\left(\log \log \gamma_{j, n_{j}}\right)^{\omega} \leq \gamma_{j, n_{j}}$, and (2) for every $k$ such that $1 \leq k<n_{j}$ and $\omega \leq \gamma_{j, k}<\left(\log \log \gamma_{j, k}\right)^{\omega}$, there is $i$ such that $k<i \leq n_{j}$ and $\gamma_{j, i}>\gamma_{j, k}$ and $\left(\log \log \gamma_{j, i}\right)^{\omega} \leq \gamma_{j, i}$.

In the interest of completeness, we give the analogue of 3.20 under the stronger hypothesis $\mathrm{SCH}$.

3.21. Theorem. Assume $\mathrm{SCH}$. For an Abelian torsion group $G$, the following conditions are equivalent.

(a) G admits a pseudocompact group topology.

(b) G has the form $G=\bigoplus_{j=1}^{m} \bigoplus_{i=1}^{n_{j}} \bigoplus_{\gamma_{j, i}} \mathbb{Z}\left(p_{j}^{r_{j, i}}\right)$ with $r_{j, 1}<\cdots<r_{j, n_{j}}$ for $1 \leq j \leq m$ and with each family $\left\{\gamma_{j, i}: 1 \leq i \leq n_{j}\right\}(1 \leq j \leq m)$ satisfying these conditions: (1) either $\gamma_{j, n_{j}}$ is finite or $\left(\log \gamma_{j, n_{j}}\right)^{\omega} \leq \gamma_{j, n_{j}}$, and (2) for every $k$ such that $1 \leq k<n_{j}$ and $\omega \leq \gamma_{j, k}<\left(\log \gamma_{j, k}\right)^{\omega}$, there is $i$ such that $k<i \leq n_{j}$ and $\gamma_{j, i}>\gamma_{j, k}$ and $\left(\log \gamma_{j, i}\right)^{\omega} \leq \gamma_{j, i}$.

Proof. Using 3.8(c) in place of 3.8(b), restate 3.18 so that (M) is replaced by $\mathrm{SCH}$ and "log $\log$ " is replaced throughout by " $\log$ ". The present statement is immediate from 3.18 (thus modified) and 3.19 .

The following question is perhaps the simplest particular case of 3.7 which we are unable to settle.

3.22. Question. Let $\gamma$ and $\delta$ be infinite cardinals, admissible and inadmissible respectively, with $\gamma>\delta$. Let $p \in \mathbb{P}$ and let $G=\bigoplus_{\gamma} \mathbb{Z}\left(p^{2}\right) \oplus \bigoplus_{\delta} \mathbb{Z}(p)$. Does $G$ satisfy $G \in \mathscr{G}(\alpha)$ for every $\alpha$ such that $\alpha \in \mathscr{A}(\gamma)$ ? In particular, does $G$ satisfy $G \in \mathscr{G}(\log \gamma)$ ?

We make three comments concerning this question. (1) From 3.11 we have either $G \in \mathscr{G}(\alpha)$, or $G \in \mathscr{G}(\kappa)$ for every $\kappa$ such that $\gamma \leq \kappa \leq 2^{\gamma}$. (2) The answer is "No" in case $\delta=\omega, \gamma=2^{\omega}$ : A pseudocompact metrizable space is compact (cf. [22] (Theorem 30), or [19] (Exercise 3D.2)), but with $\alpha=\omega$ the group $G$ admits no compact group topology (cf. [23](25.25)). (3) If $G$ is defined as above but with $\delta$ admissible, then from 3.3 follows $\bigoplus_{\delta} \mathbb{Z}(p) \in \mathscr{G}(\log \delta)$ and $\bigoplus_{\gamma} \mathbb{Z}\left(p^{2}\right) \in \mathscr{G}(\alpha)$ and hence indeed $G \in \mathscr{G}(\alpha)$.

While we have been unable to classify exactly those cardinals $\alpha$ for which a pseudocompactifiable group $G$ satisfies $G \in \mathscr{G}(\alpha)$, we achieve success (for Abelian 
torsion groups) when $\alpha \geq|G|$. As with 3.20 , our statement takes a particularly pleasing form (see 3.25) when condition (M) is assumed.

3.23. Lemma. Let $p \in \mathbb{P}$, and let $G$ be an Abelian p-group which admits a pseudocompact group topology. If $\alpha \geq \omega$ satisfies $m(\alpha) \leq|G| \leq \alpha$, then $G$ admits a pseudocompact group topology of weight $\alpha$.

Proof. $G$ has bounded order by 1.3 ; we write $G=\bigoplus_{i=1}^{n} \bigoplus_{\gamma_{i}} \mathbb{Z}\left(p^{r_{i}}\right)$ with $r_{1}<\cdots<r_{n}$. Choose any $i_{0}$ such that $\gamma_{i_{0}}=|G|$ and define $G^{\prime}=\bigoplus_{i=1}^{i_{0}} \bigoplus_{\gamma_{i}} \mathbb{Z}\left(p^{r_{i}}\right)$; further, if $i_{0}<n$, define $G^{\prime \prime}=\bigoplus_{i=i_{0}+1}^{n} \bigoplus_{\gamma_{i}} \mathbb{Z}\left(p^{r_{i}}\right)$. The condition $\gamma=2^{\alpha}$ fails, so conclusion (ii) of 3.11 fails for $G^{\prime}$. Thus (i) of 3.11 holds for $G^{\prime}$, that is, $G^{\prime}$ admits a pseudocompact group topology of weight $\alpha$. The proof is complete in case $i_{0}=n$ (since then $G^{\prime}=G$ ), so we need consider only the case $i_{0}<n$. Then $G^{\prime \prime}$ is defined, and $G^{\prime \prime}$ is an Abelian $p$-group of bounded order which satisfies the cofinal admissibility condition. Then $G^{\prime \prime}$ admits a pseudocompact group topology by 3.17 , hence by 1.2 (b) a pseudocompact group topology such that $w\left(G^{\prime \prime}\right) \leq\left|G^{\prime \prime}\right| \leq|G|$. The product topology on $G=G^{\prime} \times G^{\prime \prime}$ is as required.

3.24. Theorem. Let $G$ be an infinite Abelian torsion group and let $\alpha \geq|G|$. Then the following conditions are equivalent.

(a) $G \in \mathscr{G}(\alpha)$.

(b) $m(\alpha) \leq|G|$ and $G$ admits a pseudocompact group topology.

Proof. (a) $\Rightarrow$ (b) is clear.

(b) $\Rightarrow$ (a). First, use (a) $\Rightarrow$ (b) of 3.19 to write $G=\bigoplus_{j=1}^{m} G\left(p_{j}\right)$ where each $G\left(p_{j}\right)$ admits a pseudocompact group topology $\mathscr{T}_{j}$. By $1.2(\mathrm{~b})$ we may arrange $w\left(G\left(p_{j}\right), \mathscr{T}_{j}\right) \leq\left|G\left(p_{j}\right)\right| \leq \alpha$ for each $j$. And by 3.23 , with $j_{0}$ chosen so that $\left|G\left(p_{j_{0}}\right)\right|=|G|$, we may assume $w\left(G\left(p_{j_{0}}\right), \mathscr{T}_{j_{0}}\right)=\alpha$. Clearly the product of the topologies $\mathscr{T}_{j}(1 \leq j \leq m)$, which is pseudocompact by $1.4(\mathrm{c})$, has weight $\alpha$, as required.

As with earlier theorems, hypothesis (M) allows us to restate 3.24 using only the language of algebra and cardinal numbers.

3.25. Theorem. Assume (M), and let $G$ be an infinite Abelian torsion group with $|G| \leq \alpha$. Then $G$ admits a pseudocompact group topology of weight $\alpha$ if and only if $(\log \alpha)^{\omega} \leq|G|$ and $G$ satisfies condition (b) of Theorem 3.20.

3.26. Remark. The equivalence (a) $\Leftrightarrow$ (b) of 3.19 was announced in ZFC without proof in [14]. Using SCH, the same authors announced a characterization, similar to ours but differing in detail, of those Abelian torsion groups which admit a pseudocompact group topology. Some months after our research was completed, 
but before the present paper was submitted for publication, we heard by mail from D. Dikranjan that the characterization announced in [14] can be achieved also in ZFC, without additional axioms. He informed us that the proof of this and of other results announced in [14] and of additional related results can be found in [15], [16].

Added in proof (September 1993): In private correspondence with the authors, D. Dikranjan announced a negative answer to Question 3.7 and a positive answer to Question 3.22 for $\alpha>\omega$.

\section{References}

[1] Cater, F.S., Erdös, P., Galvin, F.: On the density of $\lambda$-box products. Gen. Top. Appl. 9 (1978), 307-312

[2] Comfort, W.W.: Topological groups. In Kenneth Kunen and Jerry E. Vaughan, editors, Handbook of Set-theoretic Topology, chapter 24, pages 1143-1263. North-Holland, Amsterdam 1984

[3] Comfort, W.W., Hofmann, K.-H., Remus, D.: Topological groups and semigroups. In M. Hušek and J. van Mill, editors, Recent Progress in General Topology, pages 59-144. Elsevier Science Publishers B.V., Amsterdam 1992

[4] Comfort, W.W., Negrepontis, S.: The Theory of Ultrafilters. Die Grundlehren der mathematischen Wissenschaften in Einzeldarstellungen, volume 221. Springer-Verlag, Berlin 1974

[5] Comfort, W.W., Remus, D.: Pseudocompact topological group topologies on Abelian groups. Abstracts Amer. Math. Soc. 12 (1991), 321

[6] Comfort, W.W., Remus, D.: Pseudocompact refinements of compact group topologies. Abstracts Amer. Math. Soc. 13 (1992), 102-103

[7] Comfort, W.W., Remus, D.: Imposing pseudocompact group topologies on Abelian groups. Fund. Math. 142 (1993), 221-240

[8] Comfort, W.W., Robertson, L.C.: Cardinality constraints for pseudocompact and for totally dense subgroups of compact Abelian groups. Pacific J. Math. 119 (1985), 265-285

[9] Comfort, W.W., Roberton, L.C.: Extremal phenomena in certain classes of totally bounded groups. Dissertationes Math. 272 (1988), 48 pages. Rozprawy Mat. Polish Scientific Publishers, Warszawa 1988

[10] Comfort, W.W., Ross, K.A.: Topologies induced by groups of characters. Fund. Math. 55 (1964), 283-291

[11] Comfort, W.W., Ross, K. A.: Pseudocompactness and uniform continuity in topological groups. Pacific J. Math. 16 (1966), 483-496

[12] Corson, H.H.: Normality in subsets of product spaces. American J. Math. 81 (1959), 785-796

[13] Devlin, K. I., Jensen, R. B.: Marginalia to a theorem of Silver. In G.H. Müller, A. Oberschelp, and K. Potthoff, editors, Proc. ISLIN Logic Conference Kiel 1974, Lecture Notes in Mathematics 499, pages 115-142. Springer-Verlag, Berlin-Heidelberg-New York 1975

[14] Dikranjan, D.N., Shakhmatov, D.B.: Pseudocompact topologizations of groups. Zbornik radova Filozofskog fakulteta u Nišu Serija Matematika 4 (1990), 83-93

[15] Dikranjan, D.N., Shakhmatov, D. B.: Algebraic structure of the pseudocompact groups, 1991. Report 91-19, pp.1-37. York University, Ontario, Canada 
[16] Dikranjan, D.N., Shakhmatov, D.B.: Algebraic structure of pseudocompact groups, $19 \infty$. Manuscript submitted for publication

[17] van Douwen, E.K.: The weight of a pseudocompact (homogeneous) space whose cardinality has countable cofinality. Proc. Amer. Math. Soc. 80 (1980), 678-682

[18] Fuchs, L.: Infinite Abelian Groups, vol. I. Academic Press, New York - San Francisco - London 1970

[19] Gillman, L., Jerison, M.: Rings of Continuous Functions. D. Van Nostrand Co., New York 1960

[20] Glicksberg, I.: Stone-Čech compactifications of products. Trans. Amer. Math. Soc. 99 (1959), 369-382

[21] Hernández, S., Sanchis, M.: $G_{\delta}$-open functionally bounded subsets in topological groups, Topology Appl. To appear

[22] Hewitt, E.: Rings of real-valued continuous functions I. Trans. Amer. Math. Soc. 64 (1948), 45-99

[23] Hewitt, E., Ross, K.A.: Abstract Harmonic Analysis, volume I. Die Grundlehren der mathematischen Wissenschaften in Einzeldarstellungen, volume 15. Springer-Verlag, Berlin-Göttingen-Heidelberg 1963

[24] Hewitt, E., Ross, K.A.: Extensions of Haar measure and of harmonic analysis for locally compact Abelian groups. Math. Ann. 160 (1965), 171-194

[25] Jech, T.: Set Theory. Academic Press, Inc., San Diego 1978

[26] Kister, J.M.: Uniform continuity and compactness in topological groups. Proc. Amer. Math. Soc. 13 (1962), 37-40

[27] Kurosch, A. G.: Gruppentheorie I. Akademie-Verlag, Berlin 1970

[28] Magidor, M.: On the singular cardinals problem I. Israel J. Math. 28 (1977), 1-31

[29] Magidor, M.: On the singular cardinals problem II. Ann. of Math. 106 (1977), 517-547

[30] Masaveu, O.: Title TBA. PhD thesis, Wesleyan University, Middletown, Connecticut, USA, $19 \infty$

[31] Weil, A.: Sur les Espaces à Structure Uniforme et sur la Topologie Générale. Publ. Math. Univ. Strasbourg. Hermann \& Cie, Paris 1937

Received October 23, 1992, in final form May 27, 1993

W.W. Comfort, Department of Mathematics, Wesleyan University, Middletown, CT 06459 USA.

EMAIL: wcomfort@eagle.wesleyan.edu. FAX: 1-203-343-3903

Dieter Remus, Institut für Mathematik, Universität Hannover, Welfengarten 1, 30167 Hannover, Germany. FAX: 49-5405-7035 
Bereitgestellt von | Technische Informationsbibliothek Hannover Angemeldet Heruntergeladen am | 27.03.18 14:50 